\title{
TRICHINELLOSIS IN FARMED WILD BOAR: MEAT INSPECTION FINDINGS AND SEROPREVALENCE
}

\author{
SUKURA A.*, NÄREAHO A.*, VEIJALAINEN P.** \& OIVANEN L.****
}

\section{Summary :}

A reflection of highly prevalent endemic wildlife trichinellosis is seen in wild boar farming in Finland. During the last five years, $0.7 \%(15 / 2265)$ of wild boars undergoing official meat inspection have been determined to be Trichinella-positive. These findings originate from six different farms. In Finland, T. spiralis and T. pseudospiralis have been discovered in meat inspection of wild boars. ELISA showed 11 out of 99 serum samples (11\%) as having specific antibodies for $T$. spiralis crude antigen. Positive samples were from three out of the thirteen farms from which the sera were available. Most of the positive serum samples (8/11) originated from a farm where trichinellosis was also revealed in meat inspection, the other two seropositive farms were without previous Trichinella records. Over the last few decades, no reports have been made of human trichinellosis acquired in Finland. This indicates both efficient meat inspection as well as public awareness of high-risk foodstuff.

KEY WORDS : ELISA, epidemiology, serology, T. spiralis, T. pseudospiralis, wild boar.

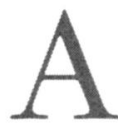
lthough wild boar farming is still a small-scale business in Finland, it has recently expanded rapidly due to a growing interest in finding alternative production lines in Finnish agriculture. All marketed wild boar meat - both game and farmed - must be officially inspected. During the last five years, the amount of inspected meat has grown 8.5 -fold, from 92 carcasses in 1995 to 786 in 1999 . Simultaneously, the number of wild boar farms has increased; according to different sources, the current number of farms is estimated at 55-80. Farming typically takes place in paddocks in forests emulating natural habitats, with only a wire-net fence segregating animals from the surroundings. Sylvatic trichinellosis is endemic in Finland, and its prevalence in wildlife in the southern part of the country is high (Oksanen \& Oivanen, 1994).

* Faculty of Veterinary Medicine, University of Helsinki, ** National Veterinary and Food Research Institute, Helsinki, Finland. Correspondence: A. Sukura.

Tel.: +358-9-19149526 - Fax: +358-9-19149799.

E-mail: antti.sukura@helsinki.fi

\section{MATERIAL AND METHODS}

$1 \sqrt{ }$ eat inspection of wild boar generally takes place in a local low-capacity slaughterhouse with traditional trichinoscopy. Alternatively, some animals are inspected in large-scale slaughterhouses that use the digestion method. All positive findings are confirmed in EELA (National Veterinary and Food Research Institute) by the digestion method. Meat-inspection statistics from EELA were used in this paper.

For molecular typing, we obtained Trichinella samples from epidemics on two farms. Both RAPD (Bandi et al., 1993) and specific primer pairs SB4-2, SB4-4 and SB5 (Wu et al., 1997) were used in PCR-assays.

Wild boar farmers in Finland have recently set up a voluntary monitoring programme for contagious swine diseases. Representative number of serum samples from slaughtered animals are sent to EELA for serological assay. Apart from the programme we analysed 99 serum samples originating from 13 different farms for Trichinella antibodies. Crude antigen of T. spiralis larval lysate was used to coat the ELISA plates. The cut-off value was set with a weakly positive serum collected during an earlier investigation from a mildly infected boar with 0.1 larvae per gram of meat (lpg).

\section{RESULTS}

D uring the last five years, Trichinella-positive wild boars have been found on six farms. Out of all inspected animals, $0.7 \%(15 / 2265)$ have been condemned due to trichinellosis. The intensity of detected infections varied from 0.07 to $680 \mathrm{lpg}$ (median $5.2 \mathrm{lpg}$ ).

On two of the positive farms, the infecting species have been identified as $T$. spiralis and $T$. pseudospiralis (Fig. 1). The first case was an outbreak where nine out of 25 slaughtered animals were positive; T. spiralis was the sole species involved (Oivanen et al., 2000). On the other farms, only sporadic cases occurred. In PCR, specific primer sets SB4-2 and SB4-4 (designed for 


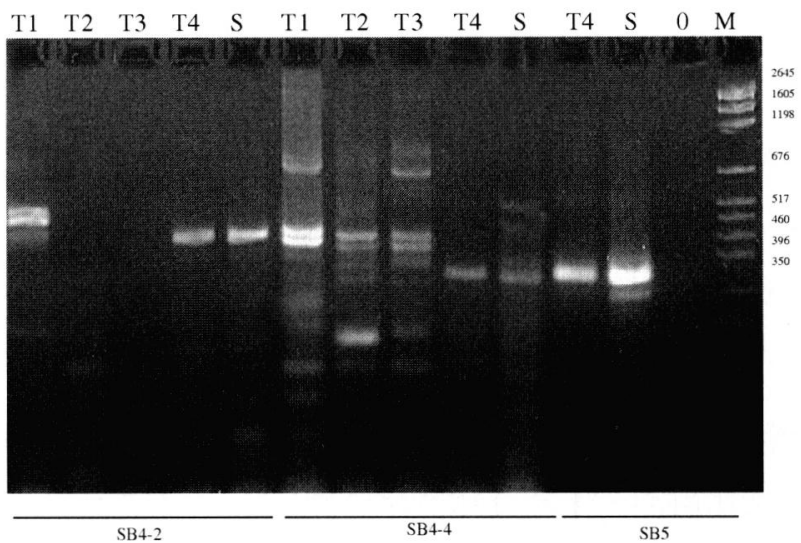

Fig. 1. - Agarose gel electrophoresis of PCR products with three primers: SB4-2, SB4-4 and SB5. T1- 4 reference strains, $T$. spiralis (ISS004), T. nativa (ISS042), T. britovi (ISS100) and T. pseudospiralis (ISS013). $\mathrm{S}$ represents sample, 0 is negative control $\mathrm{H}_{2} \mathrm{O}$, and $\mathrm{M}$ is molecular weight marker in bp.

T. spiralis) yielded band patterns in agarose gel from which species were identified by comparison with reference strains. SB5 primer pair amplified only with DNA from T. pseudospiralis (Fig. 1.). In the case of T.pseudospiralis, the original meat inspection with trichinoscopy failed to show the infection, but a suspicious consumer requested an additional examination, which was performed with digestion, revealing $11 \mathrm{lpg}$ of loin. OD-values in ELISA higher than the cut-off were seen in 11 samples in a serum dilution of 1:50. These positive samples originated from three different farms. Most of the positive samples (8/11) originated from a farm where trichinellosis had been detected during meat inspection; $32 \%(8 / 22)$ of the sera from this farm showed immunoreactivity (Fig. 2). From the two other seropositive farms, $2 / 11$ and $1 / 8$ samples were positive which could not be confirmed by meat inspection findings.

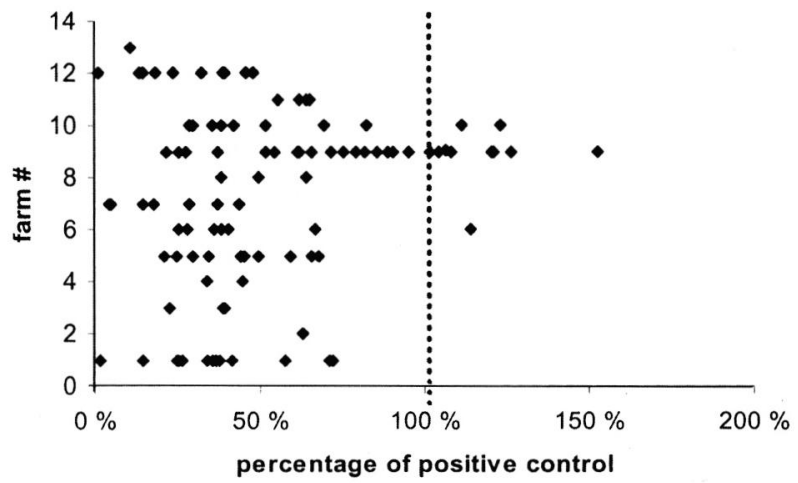

Fig. 2. - Relative ELISA-OD-values in 1:50 diluted samples from different farms. Most of the seropositive sera was concentrated at farm number 9, from which trichinellosis was also found in meat inspection. Cut-off ( $100 \%)$ of weak positive serum from mildly infected wild boar $(0.1 \mathrm{lpg})$.

\section{DISCUSSION}

T $\mathrm{n}$ Finland, trichinellosis has been detected almost annually over the last twenty years in domestic pig, but the prevalence has been low, being only $0.004 \%$ (Oivanen et al., 2000). Wild boar farming, with a corresponding prevalence of $0.7 \%$ bears a much higher risk. An obvious explanation lies in the production factors which have a closer contact to wildlife. Both T. spiralis and T. pseudospiralis have previously been reported also in feral wild boars elsewhere (PerezMartin et al., 2000, Ranque et al., 2000).

Serum samples for our survey have been obtained at slaughter in accordance with official regulations. Although we do not know exactly which serum belongs to which animal, there is good reason to believe that all serologically positive animals have passed meat inspection, with no Trichinella larvae having been found. Earlier reports have also shown circulating Trichinella antibodies with digestion-negative results.

Interestingly, a common sylvatic species in Finland, T. nativa, has not been found in farmed wild boars so far. A report of experimental infection with T. nativa in pigs failed to show muscle larvae, but did reveal high antibody response (Kapel et al., 1998). Similar results have been seen in reindeer (Oksanen et al., 2000). These and other similar reports indicate host species-specific resistance to certain Trichinella species. In Finland, two associations of wild boar farmers exist, but only the smaller one with 25 farms certifies that animals have never been cross-bred. Thus, many so-called farmed wild boars are actually cross-bred with domestic swine. Therefore, some of the seropositive results without any meat inspection findings could indicate exposure to $T$. nativa with a natural resistance of wild boar or crossbreds to the particular species. However, reports of $T$. nativa-infected sylvatic wild boar (Pozio \& Kapel, 1999) and domestic pigs (Gasser et al., 1998) do exist.

The clustering of positive serum samples to the farm with trichinellosis is intriguing. This either shows a broader exposure to Trichinella than discovered in meat inspection or indicate some factors on that farm inducing cross-reactions to yield false-positive results. Additionally, higher sensitivity of ELISA than detection of muscle larvae must be taken into consideration. Today practically all farmed wild boars are born in captivity, yet farms are not consistently Trichinella-negative. Thus, some vectors or infection routes remain to be discovered.

\section{REFERENCES}

Bandi C., la Rosa G., Comincini S., Damiani G. \& Pozio E. Random amplified polymorphic DNA technique for the identification of Trichinella species. Parasitology, 1993, 107, 419-424. 
Gasser R.B., Zhu X., Monti J.R., Dou L., Cai X. \& Pozio E. PCR-SSCP of rDNA for the identification of Trichinella isolates from mainland China. Molecular and cellular probes, 1998, 12, 27-34.

Kapel C.M.O., Webster P., Lind P., Pozio E., Henriksen S.A., MurRel K.D. \& NANSEN P. Trichinella spiralis, T. britovi, and T. nativa: infectivity, larval distribution in muscle, and antibody response after experimental infection in pigs. Parasitology Research, 1998, 84, 264-271.

Orvanen L., Mikkonen T. \& Sukura A. An outbreak of trichinellosis in farmed wild boar in Finland. APMIS. In press.

Oksanen A., Oivanen L., Eloranta E., Tirkkonen T. \& ÅsBAKK K. Experimental trichinellosis in reindeer. Journal of Parasitology, 2000, 86, 763-767.

Perez-Martin J.E., Serrano F.J., Reina D., Mora J.A. \& NavarRETE I.J. Sylvatic trichinellosis in southwestern Spain. J. Wild. Dis., 2000, 36, 531-534.

POZIO E. \& KAPEL C.M.O. Trichinella nativa in sylvatic wild boars. Journal of Helminthology, 1999, 73, 87-89.

Oivanen L. \& Oksanen A. Trichinellosis in domestic swine and wildlife in Finland. W.C. Campbell, E. Pozio and F. Bruschi (eds.) Trichinellosis. Proceedings of the Eighth International Conference on Trichinellosis. Istituto Superiore de Sanita Press, Rome, 1994: 569-574.

Ranque S., Faugere B., Pozio E., la Rosa G., Tamburrini A., Pellissier J.F. \& BrouQui P. Trichinella pseudospiralis outbreak in France. Emerging Infectious Diseases, 2000, 6, 543547.

Wu Z., Nagano I., Fukumoto S., Saito S., Yamaguchi T., PozIO E. \& TAKAHASHI Y. Polymerase chain reaction primers to identify Trichinella spiralis or T. pseudospiralis. Parasitology International, 1997, 46, 149-154. 